\title{
ICTIOFAUNA DO RIO SANTA MARIA, RS
}

I.M.K.BOSSEMEYER, M.L.C.WEIS, S.T.BENNEMANN E M.L.S.BIER

Departamento de Biologia. Centro de Ciências Naturais e Exatas.UFSM. Santa Maria, RS.

RESUMO

A fauna ictiológica do Rio Santa Maria foi pesquisada atra vés de coletas estacionais de agosto de 1984 a maio de 1985 .

são feitas consideraçoes sobre sistemática, ocorréncia e abundância das espécies coletadas.

Um total de 1345 peixes pertencentes a trinta e nove gêre ros e cinquenta e trēs espécies foram examinados e identificados.

SUMMARY

BOSSEMEYER, I.M.K.; WEIS, M.L.C.; BENNEMANN, S.T. and BIER, M.L.S., 1985. Ichthvologia fauna of the Santa Maria River, RS.Ciên cia e Natura, 7: $209-222$.

The ichthyologic fauna of the Santa Maria River was studied through seasonal collections from august 1984 to may 1985.

Considerations were made about the systematics, ocurrence and abundance of the collected species.

A total of 1345 fishes pertaining to thirty nine genera and fifty three species were examined and identified.

INTRODUÇĀO

0 estudo da distribuição e biologia das espécies de pei xes regionais tem sido alvo de pesquisas efetuadas pelo setor de Zoologia do Departamento de Biologia do Centro de Ciências Naturais e Exatas da UFSM. Durante os anos de 1982/83 efetuamos a amostragem íctica do Rio Ibicuí-Mirim. O presente trabalho encerra os resulta dos do inventārio da ictiofauna do Rio Santa Maria que, juntamente com o Rio Ibicuí-Mirim, forma o Rio Ibicuí, importante afluente do Rio Uruguai.

Todas as informaçöes obtidas nestes projetos assim como no que serā implantado no Rio Ibicuí no ano de 1985 visam, primor dialmente, afetuar o levantamento dos peixes dos rios pertencentes a Bacia do Rio Ibicuĩ objetivando estabelecer uma coleção que seja, o mais possível, representativa da fauna íctica destes importantes cursos d'ägua. E de notar que não foram realizadas coletas sistema tizadas nestes rios e que mesmo os estudos de espécies neles encon tradas demonstraram a existēncia de uma diversidade de fauna entre esta Bacia e outras do Estado.

0 Rio Santa Maria tem sua nascente na região de Torquato 
Severo, na junção da Coxilha Grande com a Serra de Santa Tecla. Sua direção inicial é SE, tomando a seguir rumo NO, com um curso de 240 KM. Tem grande extensão e largura mas sua profundidade é reduzida. Está, entretanto, sujeito a enchentes, podendo o nīvel das águas che gar entre 10 e 12 metros sobre os níveis de estiagem. As coletas ti veram lugar num ponto médio do rio, nas quatro estações do ano, no periodo de agosto de 1984 a maio de 1985. O relato da ocorréncia e da abundância das espécies coletadas objetiva a presente publicação. os aspectos biológicos ligados à alimentação serão apresentados fú turamente.

MATERIAL E METODO

A ārea de coleta constou de um trecho do Rio Santa Maria que se localiza a $15 \mathrm{~km}$ ao norte da cidade de Rosário do Sul e que se caracteriza por apresentar depósitos em barras e meandros abando nados. A margem esquerda apresenta vegetação ciliar de pequeno por te e a direita campos cultivados de arroz e soja, alternados com pe quenas extensões de mata ciliar (Figura 1).

Foram realizadas quatro coletas nas estações do ano,assim distribuídas:
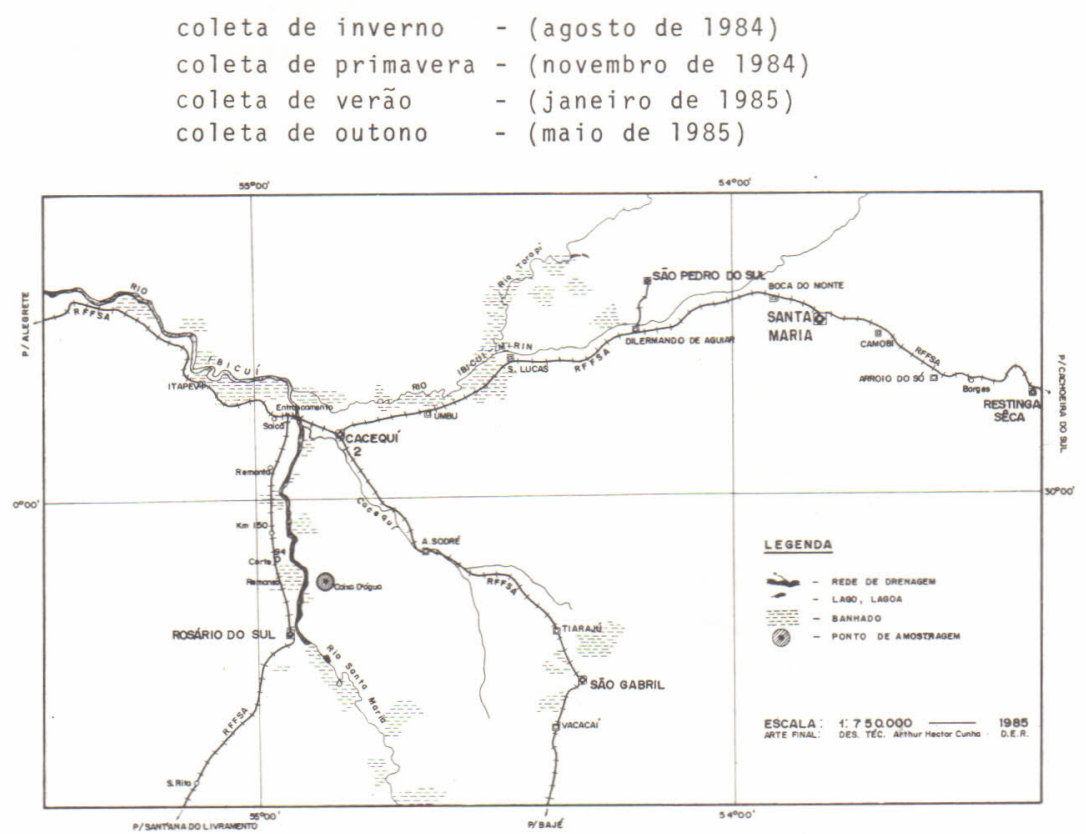

Figura 1 - Mapa de localização do ponto de coleta dos pei xes no rio Santa Maria, RS.

A técnica empregada para capturar os peixes foi a da 
utilização de redes tresmalho e de espera com malhas variando entre 1,5 e $7,0 \mathrm{~cm}$, colocadas às 17 horas e revisadas na manhã seguinte quando se efetuava a retirada dos especimens que constituiam a amos tra noturna. Ao fim da tarde recolhiam-se os peixes capturados du rante o dia, assim constituindo-se a amostra diurna. Para a captura de exemplares de pequeno porte utilizou-se o puçá.

Os exemplares que constituiam cada amostra eram medidos no seu comprimento total, pesados em gramas com aproximação até de cigramas e dissecados em campo para efetuar-se a sexagem e retirada do tubo digestivo para posterior exame do conteúdo alimentar. As amostras foram preservadas em formol a 10\% (exceto 30 exemplares de Hypostomus sp que foram devolvidos ao riol e encontram-se deposita das junto a coleção îctica deste setor. A identificação dos espec mens baseou-se na literaturá consultada $(1,2,3,4,5,7,8,9,10$, $11,12,13,14,15,16,17$, e 18).

Registrou-se o número total de exemplares por estação do ano e por amostra noturna e diurna (TABELA I e FIGURA 2).

TABELA I. NUMERO DE EXEMPLARES POR ESTAÇAOO E POR AMOSTRA NOTURNA E DIURNA.

\begin{tabular}{|c|c|c|c|}
\hline \multirow{2}{*}{$\begin{array}{l}\text { ESTAÇOES DE } \\
\text { COLETAS }\end{array}$} & \multicolumn{2}{|c|}{ NOMERO DE EXEMPLARES } & \multirow{2}{*}{ TOTAL/ESTAÇACC } \\
\hline & captura noturna & captura diurna & \\
\hline inverno & 209 & 59 & 268 \\
\hline primavera & 151 & 271 & 422 \\
\hline verão & 55 & 301 & 356 \\
\hline outono & 220 & 79 & 299 \\
\hline TOTAL/CAPTURA & 635 & 710 & 1.345 \\
\hline
\end{tabular}

Calculou-se o nümero de gêneros e espēcies por ordem e fá mỉlia bem como a distribuição percentual destas famîlias nas quatro estações do ano (TABELA II).

Computou-se, igualmente, o nümero de exemplares por espé cie, turno e sexo nas coletas anteriormente referidas (TABELAS III, IV, V e VI) e graficou-se o nūmero de exemplares daquelas espécies que foram capturadas nas quatro estações de coleta (FIGURA 3 ).

RESULTADOS

Os 1345 especimens capturados estão listados, após sua identificação, como segue:

1.1. Ordem CYPRINIFORMES

1.1.1. Subordem CHARACOIDEI 
TABELA II. NOMERO DE GENEROS E ESPECIES POR ORDENS E FAMILIAS E DISTRIBUIÇAO PERCENTUAL DESTAS FAMILIAS NAS quatro estaÇOES de COLETAS (INVERno, primavera, VERAO E OUTONO).

\begin{tabular}{|c|c|c|c|c|c|c|c|c|c|c|c|c|c|}
\hline ORDENS & FÁMILIAS & $\begin{array}{r}\text { NOM } \\
\text { GENEROS }\end{array}$ & $\begin{array}{l}\text { EROS } \\
\text { ESPECIES }\end{array}$ & Nọ $\frac{I}{E}$ & $\begin{array}{l}\text { I } \\
\text { Ex. \% }\end{array}$ & $\begin{array}{r}\quad E \\
\quad P \\
N O P \\
\end{array}$ & $\begin{array}{l}\text { ESTAÇOES } \\
\text { E } \\
\text { Ex. \% }\end{array}$ & $\begin{array}{ll}S & V \\
N O P X\end{array}$ & $\begin{array}{l}V \\
\text { Ex. \% }\end{array}$ & $\begin{array}{ll} & 0 \\
N & \text { Ex }\end{array}$ & $x . \%$ & $\begin{array}{l}\text { TOTA } \\
\text { NOQ E }\end{array}$ & $\begin{array}{l}\text { AL } \\
\text { Ex. \% }\end{array}$ \\
\hline \multirow[t]{8}{*}{ CYPRINIFORMES } & CHARACIDAE & 7 & 12 & 64 & 423,88 & 162 & 238,39 & 64 & $4 \quad 17,98$ & 83 & 27,76 & 373 & 27,73 \\
\hline & ERYTHRINIDAE & 1 & 1 & & 48,96 & 14 & $4 \quad 3,32$ & & 20,56 & 3 & 1,00 & 43 & 3,20 \\
\hline & CHARACIDIIDAE & 1 & 1 & & - & & $5 \quad 1,18$ & & $5 \quad 1,40$ & - & - & 10 & 0,74 \\
\hline & ANOSTOMIDAE & 2 & 6 & & - & 36 & 68,53 & & $4 \quad 1,12$ & 1 & 0,33 & 41 & 3,05 \\
\hline & PARODONTIDAE & 1 & 1 & & - & & - & & - & 4 & 1,34 & 4 & 0,30 \\
\hline & PROCH I LODONTIDAE & 1 & 1 & & - & & $6 \quad 1,42$ & - & - & - & - & 6 & 0,45 \\
\hline & CURIMATIDAE & 2 & 3 & 113 & 342,16 & 106 & 625,12 & 72 & 220,22 & 161 & 53,85 & 452 & 33,61 \\
\hline & CYNODONTIDAE & 1 & 1 & & - & & - & & 20,56 & 1 & 0,33 & 3 & 0,22 \\
\hline \multirow[t]{5}{*}{ SILURIFORMES } & DORADIDAE & 1 & 1 & & - & 12 & 22,84 & & 10,28 & 4 & 1,34 & 17 & 1,26 \\
\hline & AUCHENIPTERIDAE & 2 & 2 & & 10,37 & & $8 \quad 4,27$ & & 20,56 & - & - & 21 & 1,56 \\
\hline & PIMELODIDAE & 6 & 6 & 19 & 97,09 & & $8 \quad 1,90$ & 69 & 919,38 & 19 & 6,35 & 115 & 8,55 \\
\hline & CALLICHTHYDAE & 2 & 2 & & - & 14 & 43,55 & 10 & $0 \quad 2,81$ & 1 & 0,33 & 26 & 1,93 \\
\hline & LORICARIIDAE & 4 & 6 & 46 & 617,16 & 11 & 12,61 & 37 & 710,39 & 2 & 0,67 & 96 & 7,14 \\
\hline SYNBRANCHIFORMES & SYNBRANCHIDAE & 1 & 1 & & 10,37 & & - & & - & - & - & 1 & 0,07 \\
\hline CYNODONTIFORMES & POECILIIDAE & 1 & 1 & & - & & $5 \quad 1,18$ & & - & - & - & 5 & 0,37 \\
\hline ATHERINIFORMES & ATHERINIDAE & 1 & 1 & & - & & - & & - & 1 & 0,33 & 1 & 0,07 \\
\hline PLEURONECTIFORMES & ACHIRIDAE & 1 & 1 & & - & & $1 \quad 0,27$ & - & - & - & - & 1 & 0,07 \\
\hline \multirow[t]{2}{*}{ PERCIFORMES } & CICHLIDAE & 3 & 5 & & - & 17 & 74,03 & 12 & $2 \quad 3,37$ & 1 & 0,33 & 30 & 2,23 \\
\hline & SCIAENIDAE & 1 & 1 & & - & & $6 \quad 1,42$ & 76 & 621,35 & 18 & 6,02 & 100 & 7,43 \\
\hline TOTAL & & 39 & 53 & 268 & & 422 & & 356 & & 299 & & 1.345 & \\
\hline
\end{tabular}

$I=$ inverno

$P=$ primavera

$V=$ veräo

$0=$ outono 


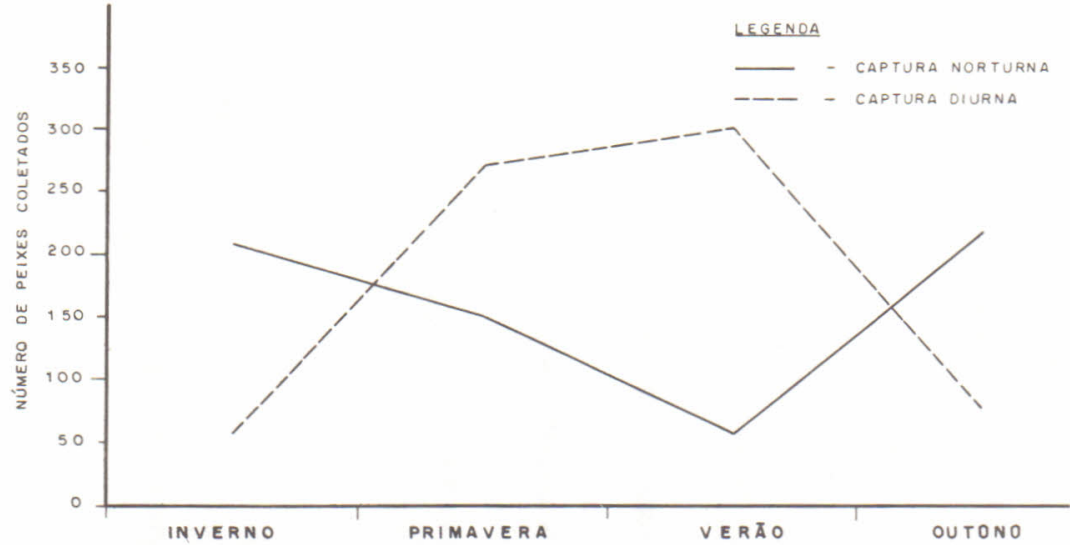

ESTACÖES DE COLETAS

Figura 2 - Nümero de peixes coletados em capturas norturna e diurna, por estação.

TABELA III. NUMERO DE EXEMPLARES CAPTURADOS NO RIO SANTA MARIA, RS, POR ESPECIE, TURNO E SEXO, DURANTE A 1 a coleta (INVERNo).

\begin{tabular}{|c|c|c|c|c|c|c|}
\hline \multirow{2}{*}{ ESPECIE } & \multirow{2}{*}{$\begin{array}{l}\text { NO EXEM } \\
\text { PLARES. }\end{array}$} & \multicolumn{2}{|c|}{ COLETA } & \multicolumn{2}{|c|}{ SEXO } & \\
\hline & & NOTURNA & DIURNA & MACHO & FEMEA & \\
\hline cynopotamus argenteus & 1 & - & 1 & 1 & - & \\
\hline Acestrorhynchus altus & 4 & - & 4 & 2 & 2 & \\
\hline oligosareus jenynsii & 12 & 12 & - & 3 & 5 & \\
\hline oligosareus hepsetus & 23 & 23 & - & 6 & 11 & \\
\hline Astyanax bimaculatus & 4 & 4 & - & 1 & 1 & \\
\hline Astyanax fasciatus & 20 & 18 & 2 & 3 & 10 & \\
\hline Hoplias malabaricus & 24 & 22 & 2 & 10 & 13 & \\
\hline Pseudocurimata gilberti & 52 & 13 & 39 & 30 & 8 & \\
\hline Pseudocurimata sp & 41 & 33 & 8 & 19 & 7 & 15 \\
\hline Curimata sp & 20 & 20 & - & 6 & 7 & \\
\hline Auchenipterus $s p$ & 1 & 1 & - & - & 1 & \\
\hline Pimelodus maculatus & 1 & 1 & - & 1 & - & \\
\hline Iheringichthys labrosus & 6 & 6 & - & - & 6 & \\
\hline Rhamdia (Tipo II) & 12 & 12 & - & 7 & 5 & \\
\hline Loricarichthys anus & 6 & 5 & 1 & 2 & 3 & \\
\hline Hipostomus $s p$ & 40 & 38 & 2 & 3 & 7 & $30 \mathrm{D}$ \\
\hline Symbranchus marmoratus & 1 & 1 & - & - & - & \\
\hline TOTAIS & 268 & 209 & 59 & 94 & 86 & 8 \\
\hline
\end{tabular}

D - devolvidos ao rio

I - sexo indeterminado 


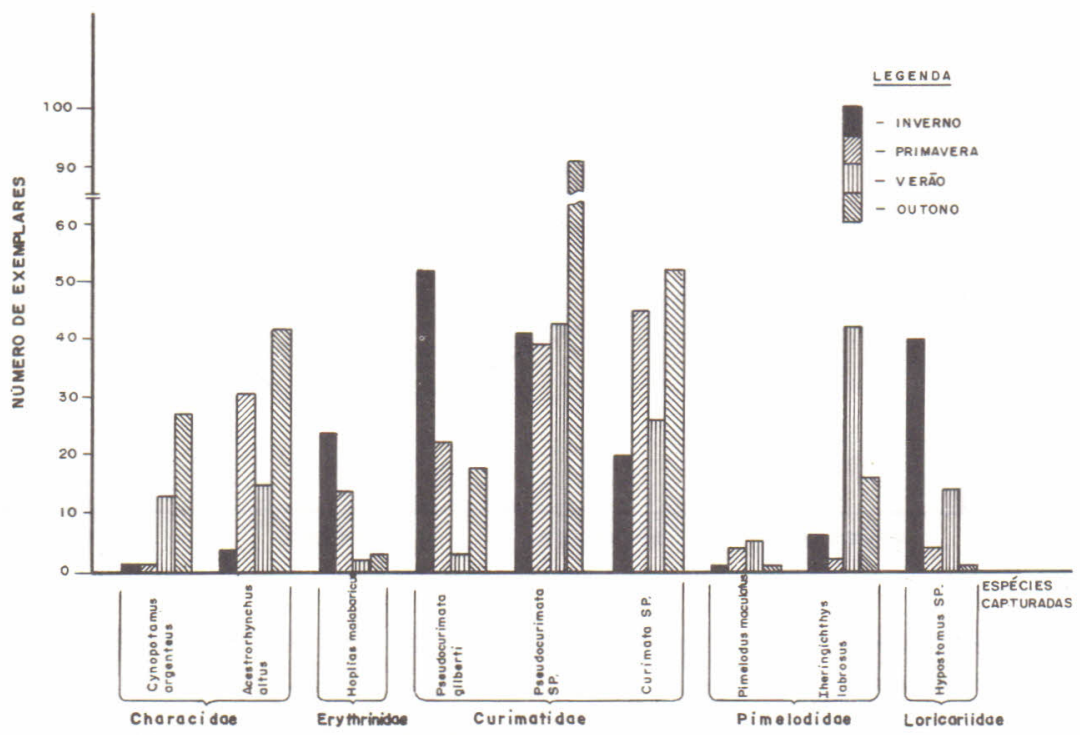

Figura 3 - Número de exemplares por famỉlias e espécies capturadas nas quatro estações de coletas.

A) Familia CHARACIDAE
a) Subfamilia CHARACINAE
Nome Comum
Cynopotamus argenteus (Valenciennes, 1847) Peixe-cachorro
b) Subfamîlia ACESTRORHYNCHINAE
Acestrorhynchus altus Menezes, 1969 Tambicu
oligosarcus jenynsii (Gunther, 1864) Tambicu
Oligosarcus hepsetus (Cuvier, 1829) Tambicu
c) Subfamilia TETRAGONOPTERINAE
Astyanax (Poecilurichthys) bimaculatus (Linnē,1758) Lambari
Astyanax (Astyanax) fasciatus(Cuvier, 1819) Lambari
Astyanax sp
Lambari
d) Subfamilia CHEIRODONTINAE
Cheirodon interruptus (Jenyns, 1842) Lambari
Cheirodon piaba Lutken, 1874 Lambari
Cheirodon $s p$ Lambari
Aphyocharax anisitsi Eigenmann \& Kennedy, 1903 Lambari
e) Subfamilia SERRASALMINAE
Serrasatmus $s p \quad$ Palometa ou piranha
OBS.: Há possivelmente mais de uma espécie no gênero Serrasalmus Lacépēde, 1803. Nosso material está presentemente sendo estudado por alguns de nós (M.L.C.W. e I.M.K.B.) 
TABELA IV. NOMERO DE EXEMPLARES CAPTURADOS NO RIO SANTA MARIA, RS, POR ESPECIE, TURNO E SEXO, DURANTE A $2 \stackrel{\text { a COLETA (PRIMAVE }}{\text { C }}$ $R A)$.

\begin{tabular}{|c|c|c|c|c|c|c|}
\hline \multirow{2}{*}{ ESPECIE } & \multirow{2}{*}{$\begin{array}{l}\text { Nọ EXEM } \\
\text { PLARES }\end{array}$} & \multicolumn{2}{|c|}{ COLETA } & \multicolumn{2}{|c|}{ SEXO } & \multirow[b]{2}{*}{ I } \\
\hline & & NOTURNA & DIURNA & MACHO & FEMEA & \\
\hline Cynopotamus argenteus & 1 & 1 & - & - & 1 & - \\
\hline Acestrorhynchus altus & 31 & 14 & 17 & 10 & 20 & 1 \\
\hline oligosareus jenynsii & 1 & 1 & - & - & 1 & - \\
\hline Astyanax bimaculatus & 17 & 4 & 13 & 3 & 9 & 5 \\
\hline Astyanax fasciatus & 22 & - & 22 & 5 & 8 & 9 \\
\hline Astyanax $s p$ & 6 & - & 6 & - & - & 6 \\
\hline Cheirodon interruptus & 8 & - & 8 & - & - & 8 \\
\hline Cheirodon piaba & 59 & - & 59 & - & - & 59 \\
\hline Cheirodon $s p$ & 17 & - & 17 & - & - & 17 \\
\hline Hoplias malabaricus & 14 & 10 & 4 & 5 & 9 & 1 \\
\hline Characidium fasciatum & 5 & - & 5 & - & - & 5 \\
\hline Schizodon platae & 30 & 24 & 6 & 4 & 22 & 4 \\
\hline Schizodon nasutus & 1 & - & 1 & 1 & - & - \\
\hline Leporinus pachyurus & 1 & 1 & - & 1 & - & - \\
\hline Leporinus sp. & 3 & 2 & 1 & - & 3 & - \\
\hline Leporinus striatus & 1 & - & 1 & - & 1 & - \\
\hline Prochilodus serofa & 6 & 6 & - & 2 & 3 & 1 \\
\hline Pseudocurimata gizberti & 22 & 7 & 15 & 8 & 5 & 9 \\
\hline Pseudocurimata $s p$ & 39 & - & 39 & 22 & 4 & 13 \\
\hline Curimata $s p$ & 45 & 23 & 22 & 12 & 19 & 14 \\
\hline Rhinodotas d'orbignyi & 12 & 12 & - & 4 & 8 & - \\
\hline Parauchenipterus galeatus & 18 & 18 & - & 8 & 10 & - \\
\hline Pimelodus maculatus & 4 & 4 & - & - & 4 & - \\
\hline Parapimelodus valeneiennesi & 1 & 1 & - & - & - & 1 \\
\hline Iheringivhthys labrosus & 2 & 1 & 1 & - & 1 & - \\
\hline Rhamdia (Tipo II) & 1 & 1 & - & 1 & - & - \\
\hline Hoplosternum littorale & 9 & 8 & 1 & 7 & 2 & - \\
\hline Corydoras paleatus & 6 & - & 6 & - & - & 6 \\
\hline Loricariichthys anus & 4 & 4 & - & 2 & 2 & - \\
\hline Loricariichthys labialis & 1 & 1 & - & - & 1 & - \\
\hline Loricarrichthys typus & 1 & - & 1 & - & - & 1 \\
\hline Hypostomus $s p$ & 4 & 3 & 1 & 2 & 1 & 1 \\
\hline Rhinelepis aspera & 1 & 1 & - & 1 & - & - \\
\hline Phalloceros caudimaculatus & 5 & - & 5 & - & - & 5 \\
\hline Achirus sp & 1 & 1 & - & - & 1 & - \\
\hline Cichlaurus facetus & 2 & 2 & - & 1 & 1 & - \\
\hline Aequidens portalegrensis & 13 & - & 13 & - & - & 13 \\
\hline Crenicichla lepidota & 1 & - & 1 & - & - & 1 \\
\hline Crenicichla saxatilis & 1 & - & 1 & 1 & - & - \\
\hline Pachyurus bonariensis & 6 & 1 & 5 & - & 6 & - \\
\hline TOTAIS & 422 & 151 & 271 & 100 & 142 & 180 \\
\hline
\end{tabular}


TABELA V. NUMERO DE EXEMPLARES CAPTURADOS NO RIO SANTA MARIA, RS, POR ESPECIE, TURNO E SEXO, DURANTE A 3 a COLETA (VERAO).

\begin{tabular}{|c|c|c|c|c|c|c|}
\hline \multirow{2}{*}{ ESPECIE } & \multirow{2}{*}{$\begin{array}{l}\text { NO EXEM } \\
\text { PLARES }\end{array}$} & \multicolumn{2}{|c|}{ COLETA } & \multicolumn{2}{|c|}{ SEXO } & \multirow[b]{2}{*}{ I } \\
\hline & & NOTURNA & DIURNA & MACHO & FEMEA & \\
\hline Cynopotamus argenteus & 13 & 4 & 9 & 4 & 8 & - \\
\hline Acestrophynchus altus & 15 & 1 & 14 & 6 & 9 & - \\
\hline oligosarcus hepsetus & 3 & - & 3 & - & - & 3 \\
\hline Astyanax bimaculatus & 21 & - & 21 & 7 & 8 & 6 \\
\hline Aphyocharax anisitsi & 3 & - & 3 & - & - & 3 \\
\hline Serrasalmus $s p$ & 9 & - & 9 & - & - & 9 \\
\hline Hoplias malabaricus & 2 & 2 & - & - & 2 & - \\
\hline Characidium fasciatum & 5 & - & 5 & - & - & 5 \\
\hline Schizodon platae & 1 & 1 & - & - & 1 & - \\
\hline Schizodon nasutus & 1 & - & 1 & - & 1 & - \\
\hline Leporinus obtusidens & 1 & - & 1 & - & 1 & - \\
\hline Leporinus striatus & 1 & - & 1 & - & 1 & - \\
\hline Pseudocurimata gilberti & 3 & - & 3 & - & - & 3 \\
\hline Pseudocurimata $s p$ & 43 & - & 43 & 25 & 18 & - \\
\hline Curimata sp & 26 & - & 26 & 11 & 6 & 9 \\
\hline Rhaphiodon vulpinus & 2 & 1 & 1 & - & 2 & - \\
\hline Rhinodoras d'orbignyi & 1 & - & 1 & - & 1 & - \\
\hline Auchenipterus sp & 2 & - & 2 & 2 & - & - \\
\hline Pimezodus maculatus & 5 & 1 & 4 & 2 & 3 & - \\
\hline Parapimelodus valenciennesi & 18 & 2 & 16 & 5 & 13 & - \\
\hline Iheringichthys labrosus & 42 & 1 & 41 & 3 & 14 & 25 \\
\hline Rhamdia (Tipo II) & 2 & 1 & 1 & - & 2 & - \\
\hline Sorubim lima & 2 & 1 & 1 & 1 & 1 & - \\
\hline Hoplosternum littorale & 1 & - & 1 & 1 & - & - \\
\hline Corydoras paleatus & 9 & - & 9 & - & - & 9 \\
\hline Loricarichthys anus & 3 & - & 3 & 2 & 1 & - \\
\hline Loricarichthys labialis & 11 & 11 & - & - & 9 & 2 \\
\hline Loricariichthys typus & 6 & 6 & - & - & - & 6 \\
\hline Loricaria $s p$ & 3 & 1 & 2 & 1 & 2 & - \\
\hline Hypostomus sp & 14 & 14 & - & 7 & 7 & - \\
\hline Cichlaurus facetus & 3 & - & 3 & 3 & - & - \\
\hline Aequidens portalegrensis & 3 & - & 3 & - & - & - \\
\hline Crenicichla vittata & 6 & 1 & 5 & 3 & 2 & 1 \\
\hline Pachyurus bonariensis & 76 & 7 & 69 & 9 & 15 & 52 \\
\hline TOTAIS & 356 & 55 & 301 & 92 & 127 & 137 \\
\hline
\end{tabular}


TABELA VI. NOMERO DE EXEMPLARES CAPTURADOS NO RIO SANTA MARIA, RS, POR ESPECIE, TURNO E SEXO, DURANTE A 4 a COLETA (OUTONO).

\begin{tabular}{|c|c|c|c|c|c|c|}
\hline & \multirow{2}{*}{$\begin{array}{l}\text { NO EXEM } \\
\text { PLARES }\end{array}$} & \multicolumn{2}{|c|}{ COLETA } & \multicolumn{2}{|c|}{ SEXO } & \multirow[b]{2}{*}{ I } \\
\hline & & NOTURNA & DIURNA & MACHO & FEMEA & \\
\hline Cynopotamus argenteus & 27 & 27 & - & 18 & 8 & - \\
\hline Acestrorhynchus altus & 42 & 17 & 25 & 13 & 28 & - \\
\hline oligosarcus jenynsii & 5 & 5 & - & - & 5 & - \\
\hline Astyanax fasciatus & 5 & 5 & - & - & - & 5 \\
\hline Astyanax sp & 2 & 2 & - & - & - & 2 \\
\hline Serrasalmus sp & 2 & - & 2 & - & - & 2 \\
\hline Hoplias malabaricus & 3 & 3 & - & 2 & 1 & - \\
\hline Schizodon platae & 1 & 1 & - & - & 1 & - \\
\hline Apareiodon affinis & 4 & - & 4 & - & - & 4 \\
\hline Pseudocurimata gilberti & 18 & 18 & - & 10 & 8 & - \\
\hline Pseudocurimata sp & 91 & 66 & 25 & 8 & 46 & 7 \\
\hline Curimata sp & 52 & 52 & - & 23 & 29 & - \\
\hline Rhaphiodon vulpinus & 1 & 1 & - & 1 & - & - \\
\hline Rhinodoras d'orbignyi & 4 & 4 & - & - & 4 & - \\
\hline Pimerodus maculatus & 1 & 1 & - & - & 1 & - \\
\hline Pimerodelia sp & 2 & - & 2 & - & - & 2 \\
\hline Iheringichthys Zabrosus & 16 & - & 16 & - & - & 16 \\
\hline Hoplosternum littorale & 1 & - & 1 & - & - & 1 \\
\hline Loricariichthys labialis & 1 & - & 1 & - & 1 & - \\
\hline Hypostomus sp & 1 & 1 & - & 1 & - & - \\
\hline Odontesthes $s p$ & 1 & 1 & - & - & 1 & - \\
\hline Crenicichla vittata & 1 & - & 1 & - & - & 1 \\
\hline Pachyurus bonariensis & 18 & 16 & 2 & 10 & 8 & - \\
\hline TOTA IS & 299 & 220 & 79 & 87 & 142 & 70 \\
\hline
\end{tabular}

B) Familia ERYTHRINIDAE

Hoplias malabaricus (Bloch, 1794)

Nome Comum

Traira

C) Familia CHARACIDIIDAE

Characidium fasciatus Reinhardt, 1866

Canivete ou

Torpedo

D) Famĩlia ANOSTOMIDAE

Schizodon platae (Garman, 1890)

Voga

Schizodon nasutus Kner, 1859

Voga

OBS.: As espécies representantes do gēnero Schizodon, cons tantes em nossa coleção, foram recentemente revistas por BENNEMANN (1) o que levou à identificação das duas espécies acima referidas. S.platae às vezes é con fundida com S.fasciatus por possuirem algumas cara $\bar{c}$ terísticas similares. 
Leporinus pachyurus Cuvier e Valenciennes, 1849 Piava

Leporinus obtusidens(Valenciennes 1847) Piava

Leporinus sp Campos, 1944

Piavinha

OBS.: Esta espécie está sendo objeto de estudo pelos auto res, uma vez que há possibilidade de ser uma espēcie não citada para o Rio Grande do Sul.

Leporinus striatus Kner, 1859

Canivete

E) Família PARODONTIDAE

Apareiodon affinis (Steindachner, 1879) Canivete

F) Familia PROCHILODONTIDAE

Prochizodus scrofa Steindachner, 1882

Grumatã

OBS.: 0 exemplar de maior peso (2.570 gramas) capturado du rante as coletas, pertence a esta espécie.

G) Família CURIMATIDAE

Pseudocurimata gilberti (Quoy \& Gaimard, 1824) Biru

OBS.: Esta espécie confere com Pseudocurimata sp Tipo A, da publicação de WEIS (19).

Curimata sp

Biru

Pseudocurimata $\mathrm{sp}$

Biru

OBS.: Foi o mais abundante dos peixes capturados, perfa zendo $15,91 \%$ do total dos especimes coletados. Este biru se caracteriza pela presença de um sinal = re petido em cada escama da linha lateral.

H) Família CYNODONTIDAE

Rhaphiodon vulpinus Agassiz, 1829

Peixe-ca

chorro

OBS.: O maior exemplar capturado durante as coletas perten ce a esta espēcie (56 cm).

1.2. Ordem SILURIFORMES

1.2.1. Subordem SILUROIDEI

A) Familia DORADIDAE

Rhinodoras d'orbignyi (Kryyer, 1855) Armado

B) Famili ia AUCHEN IPTERIDAE

Parauchenipterus galeatus (Linnaeus, 1766) Cangati

Auchenipterus sp

Bagrezinho

B) Familia PIMELODIDAE

a) Subfamỉia PIMELODINAE

$\begin{array}{ll}\text { Pimelodus maculatus (Lacēpēde, 1803) } & \text { Pintado } \\ \text { Parapimeindus valenciennesi (Krbyer, 1874) } & \text { Mandi } \\ \text { Pimelodelta sp } & \text { Porrudo ou Mandi }\end{array}$




\section{Ihemingichthys Zabrosus (Kryyer, 1855) Bagre ou pin \\ Rhamdia (Tipo II)}

b) Subfamilia SORUBIMINAE

Sorubim lima (Schneider, 1801)

D) Famîlia CALLICHTHYIDAE

Hoplostemum Zittorale (Hancock, 1828)

Corydoras paleatus (Jenyns, 1842)

E) Famīi ia LORICARIIDAE

a) Subfamilia LORICARIINAE

Loricarichthys anus Valenciennes, 1840

Cascudo viola

Loricarichthys Zabialis Boulenger, 1895

Cascudo viola

Loricariichthys typus (Blecker, 1862)

Loricamia sp

Cascudo viola

Cascudo viola

b) Subfamîlia NEOPLECOSTOMINAE

Rhinelepis aspera Spix, 1829

Cascudo preto

c) Subfamīia HYPOSTOMINAE

Hypostomus sp

Cascudo

OBS.: Gēnero mais abundante entre os Siluriformes.

2. Superordem ACANTHOPTERYGII

2.1. Ordem SYMBRANCHIFORMES

2.1.1. Subordem SYMBRANCHOIDEI

Famïlia SYMBRANCHIDAE

Symbranchus marmoratue Bloch, 1795 .Muçum

2.2. Ordem CYPRINODONTIFORMES

2.2.1. Subordem CYPRINODONTOIDEI

Famīia POECILIIDAE

Subfamīlia POECIL I INAE

Phalloceros caudimaculatue (Hense1, 1868) Barrigudinho

2.3. Ordem ATHERINIFORMES

2.3.1. Subordem ATHERINOIDEI

Famĩlia ATHERINIDAE

odontesthes $s p$

Peixe-rei

2.4. Ordem PLEURONECTIFORMES

2.4.1.. Subordem SOLEOIDEI

Famili ia ACHIRIDAE

Achirus sp

Linguado

2.5. Ordem PERCIFORMES

2.5.1. Subordem PERCOIDEI 
A) Familia CICHLIDAE

Cichlaurus facetus (Jenyns, 1842)

Carā

Aequidens portalegrensis (Hense 1, 1870)

Carā

Crenicichla lepidota Hecke1, 1840

Joana ou Joaninha

Crenicichla vittata Hecke1, 1840

Joana ou Joaninha

Crenicichla saxatilis (Linné, 1758)

Joana ou Joaninha

B) Familia SCIAENIDAE

Pachyurus bonariensis Steindachner, 1879 Corvina

CONSIDERAÇOES FINAIS

Nas coletas de inverno, primavera e outono, o nível do rio apresentou-se acima do normal e no verão foi de estiagem.

Dos 1345 exemplares coletados, 710 foram capturados no periodo diurno e 635 no período noturno. As capturas noturnas est $\underline{i}$ veram melhor representadas no inverno e outono, enquanto que as cap turas diurnas tiveram mais sucesso na primavera e verão (TABELA I FIGURA 2).

0s exemplares coletados foram enquadrados em sete ordens, dezenove famīlias, trinta e nove gēneros e cinquenta e três espécies. As ordens Cypriniformes e siluriformes, como era esperado, apresen taram o maior nūmero de famīlias, gēneros e espécies, perfazendo $69,30 \%$ e $20,45 \%$ respectivamente, do total dos especimes coletados; as demais ordens complementaram os $10,25 \%$ restantes.

A familia Curimatidae foi a melhor representada quantita tivamente com 452 exempiares, seguida pela familia Characidae, com 373 exemplares, ambas perfazendo $61,38 \%$ do total dos peixes coleta dos. Dentre os siluriformes, 211 exemplares ou seja $15,70 \%$ do total pertencem as familias Pimelodidae e Loricariidae (TABELA II).

Das dezenove famīias, apenas cinco estiveram presentes nas quatro coletas: Characidae, com Cynopotamus argenteus e Aces trorhynchus altus; Erythrinidae, com Hoplias malabaricus; Curimati dae com Pseudocurimata gilberti, Pseudocurimata sp e Curimata sp; Pimelodidae com Pimelodus maculatus e Iheringichthys labrosus e Lo ricariidae com Hypostomus sp. Destas sete espécies a de maior fre quencia no total das quatro coletas foi Pseudocurimata sp, embora os demais curimatídeos, especialmente a espécie Curimata sp, tives sem ocorrência bastante expressiva. A espécie Pimelodus maculatus mesmo estando presente em todas as estações, registrou um número muito restrito de exemplares (FIGURA 3 ).

Dos 870 especimens sexados, $42,87 \%$ pertencem ao sexo mas culino e $57,13 \%$ ao sexo feminino (TABELAS III, IV, V e VI).

\section{AGRADECIMENTOS}

Ao Dr. Naércio A. Menezes do Museu de Zoologia da U.S.P. 
pela identificação das espēcies Parauchenipterus galeatus, Acestrorhyn chus altus e Pachyurus bonariensis e ao Conselho Nacional de Desen volvimento Científico e Tecnolögico (CNPq) pelo suporte financeiro para a execução deste trabalho.

\section{REFERENCIAS BIBLIOGRĀFICAS}

1. BENNEMANN, S.T. \& BOSSEMEYER, I.M.K. Aspectos da sistemätica de Schizodon nasutus e Schizodon platae. Revista Centro de Ciên cias Rurais, Santa Maria, 15(3):271-289, 1985.

2. BRITSKI, H. Peixes de àgua doce no Estado de São Paulo - Siste mātica. In: COMISSÃo INTERESTADUAL DA BACIA PARANÁ - URUGUAI. Poluição e Piscicultura. São Paulo, Faculdade de Saūde Pūblị ca da USP e Inst. de Pesca, CPRN, S.A., 1970, 79-108 p.

3. DEVINCENZI, C.J. \& TEAGUE, G.W. Ictiofauna del Rio Uruguay Mé dio. Anales del Museo de Hist. Nat., Montevidēo, 5 (4):1-109, 1942 .

4. EIGENMANN, C.H.\& EIGENMANN, R.S. Preliminary notes on South America Nematognathi. Proc. California Acad.Sci., 2(1):119-79, 1888 .

5. EIGENMANN, C.H. \& BRAY, W.L. A revision of the american Cichli dae. Anals N.Y.Acad.Sei., VII:607-24, 1894.

6. FORTES, A.B. Geografia Fisica do Rio Grande do Sul. Porto Ale gre, Globo, 1959, 393 p.

7. FOWLER, H.W. Os peixes de ägua doce do Brasil. Arq. de Zoologia do Est. de São Paulo, São Paulo, VI:1-204, 1948.

8. Os peixes de ägua doce do Brasil. Arq. de Zoologia do Est. de São Paulo, São Paulo, VI:205-404, 1950.

9. Os peixes de āgua doce do Brasil. Arq. de Zoologia do Est. de São Paulo, São Paulo, VI: 405-628, 1951.

10. Os peixes de āgua doce do Brasil. Arq. de Zoologia do Est. de São Paulo, São Paulo, IX:1-400, 1954.

11. GARAVELLO, J.C. Revisão taxonömica do gênero Leporinus Spix, 1829. São Paulo, 415 p. Departamento Zoologia da USP. (Tese de Doutorado, 1979.

12. GERY, J. Characoids of the Word, USA, TFH Publications. Inc. Ltd., 1977. 632 p.

13. GOSLINE, W.A. Contribuitions to the classification of the Lori cariid catfishes. Ar.Mus.Nac., Rio de Janeiro, 41:79-134, 1947.

14. GUEDES, D. de S. Contribuição ao estudo da sistemática e alimen tacão de jundiás (Rhamdia spp) na região central do Rio Gran de do Suz (Pisces, Pimelodidae). Santa Maria, 95p. Disserta ção (Mestr. Zootecnia). Universidade Federal de Santa Maria, Santa Maria, 1980.

15. MENEZES, N.A. Systematics and evolution of the tribe Acestrorhyncinii 
(Pisces, Characidae). Arq. de Zoologia, São Paulo, 18(1-2): $1-150,1969$.

16. MENEZES, N.A. On the Cynopotamine, a new subfamily of Characidae (Osteichthyes, Ostariophysi, Characoidei). Arq. de Zoologia, São Pau10, 28(2):1-91, 1976.

17. RIBEIR0, A. de M. "Fauna Brasiliense" - Peixes (Eleutherobran chios-Aspirophoros) - Physoclisti. Arq.Mus.Nac., Rio de Ja neiro, 17:1-827, 1915 .

18. RINGUELET, R.A.; ARAMBURU, R.H. \& ARAMBURU, A.A. Los peces argen tinos de agua dulce. La Plata, Librart, 1967, 602 p.

19. WEIS, M.L.C.; BOSSEMEYER, I.M.K.; BIER, M.L.S. \& LIPPOLD, H.O. Inventārio da fauna ictiolōgica do Rio Ibicuí-Mirim, RS.Ciên cia e Natura, Santa Maria, 5:135-152, 1983.

Recebido em outubro, 1985; aceito em novembro, 1985. 